\title{
Astrocytes Promote Oligodendrogenesis after White Matter Damage via Brain-Derived Neurotrophic Factor
}

\author{
Nobukazu Miyamoto, ${ }^{1 \star}$ Takakuni Maki, ${ }^{1 \star}$ (ํ) Akihiro Shindo, ${ }^{1}$ Anna C. Liang, ${ }^{1}$ Mitsuyo Maeda, ${ }^{3}$ Naohiro Egawa, ${ }^{1}$ \\ Kanako Itoh, ${ }^{1}$ Evan K. Lo, ${ }^{1}$ Josephine Lok, ${ }^{1,2}{ }^{\circledR}$ Masafumi Ihara, ${ }^{4}$ and Ken Arai ${ }^{1}$ \\ ${ }^{1}$ Neuroprotection Research Laboratory, Departments of Radiology and Neurology, and ${ }^{2}$ Department of Pediatrics, Massachusetts General Hospital and \\ Harvard Medical School, Boston, Massachusetts 02114, ${ }^{3}$ Multi-Modal Microstructure Analysis Unit, RIKEN CLST-JEOL Collaboration Center, RIKEN \\ Center for Life Science Technologies, Kobe, Japan 650-0047, and ${ }^{4}$ Department of Stroke and Cerebrovascular Diseases, National Cerebral and \\ Cardiovascular Center, Suita Osaka, Japan 565-8565
}

Oligodendrocyte precursor cells (OPCs) in the adult brain contribute to white matter homeostasis. After white matter damage, OPCs compensate for oligodendrocyte loss by differentiating into mature oligodendrocytes. However, the underlying mechanisms remain to be fully defined. Here, we test the hypothesis that, during endogenous recovery from white matter ischemic injury, astrocytes support the maturation of OPCs by secreting brain-derived neurotrophic factor (BDNF). For in vitro experiments, cultured primary OPCs and astrocytes were prepared from postnatal day 2 rat cortex. When OPCs were subjected to chemical hypoxic stress by exposing them to sublethal $\mathrm{CoCl}_{2}$ for $7 \mathrm{~d}$, in vitro OPC differentiation into oligodendrocytes was significantly suppressed. Conditioned medium from astrocytes (astro-medium) restored the process of OPC maturation even under the stressed conditions. When astro-medium was filtered with TrkB-Fc to remove BDNF, the BDNF-deficient astro-medium no longer supported OPC maturation. For in vivo experiments, we

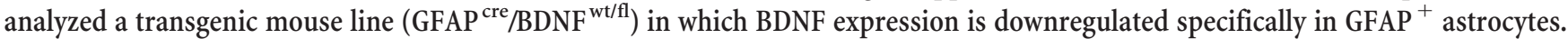
Both wild-type $\left(\mathrm{GFAP}^{\mathrm{wt}} / \mathrm{BDNF}^{\mathrm{wt} / \mathrm{fl}}\right.$ mice) and transgenic mice were subjected to prolonged cerebral hypoperfusion by bilateral common carotid artery stenosis. As expected, compared with wild-type mice, the transgenic mice exhibited a lower number of newly generated oligodendrocytes and larger white matter damage. Together, these findings demonstrate that, during endogenous recovery from white matter damage, astrocytes may promote oligodendrogenesis by secreting BDNF.

Key words: astrocyte; BDNF; oligodendrogenesis; prolonged cerebral hypoperfusion; white matter injury

Significance Statement

The repair of white matter after brain injury and neurodegeneration remains a tremendous hurdle for a wide spectrum of CNS disorders. One potentially important opportunity may reside in the response of residual oligodendrocyte precursor cells (OPCs). OPCs may serve as a back-up for generating mature oligodendrocytes in damaged white matter. However, the underlying mechanisms are still mostly unknown. Here, we use a combination of cell biology and an animal model to report a new pathway in which astrocyte-derived BDNF supports oligodendrogenesis and regeneration after white matter damage. These findings provide new mechanistic insight into white matter physiology and pathophysiology, which would be broadly and clinically applicable to CNS disease.

\section{Introduction}

Oligodendrocyte precursor cells (OPCs) are immature forms of oligodendrocytes that are the key source of myelin production.

\footnotetext{
Received April 24, 2015; revised Aug. 31, 2015; accepted Sept. 8, 2015.

Author contributions: N.M., T.M., J.L., M.I., and K.A. designed research; N.M., T.M., A.S., A.C.L., M.M., N.E., K.I., and E.K.L. performed research; M.I. contributed unpublished reagents/analytic tools; N.M., T.M., A.S., A.C.L., M.M., N.E., K.I., E.K.L., and K.A. analyzed data; N.M., J.L., and K.A. wrote the paper.

This work was supported in part by a National Institutes of Health Research Abroad Grant from the Uehara Memorial Foundation.

The authors declare no competing financial interests.

*N.M. and T.M. contributed equally to this work.
}

Although most OPCs differentiate into mature myelinating oligodendrocytes during embryonic development, a significant number of them remain in an immature state in the adult brain, making up 5-8\% of the glial cell population in the CNS (Levine et al., 2001; Dawson et al., 2003). These residual OPCs contribute to myelin sheath renewal by differentiating into oligodendrocytes throughout adult life as part of normal maintenance.

Correspondence should be addressed to Ken Arai, Neuroprotection Research Laboratory, MGH East 149-2401 Charlestown, MA 02129. E-mail: karai@partners.org.

DOI:10.1523/JNEUROSCI.1592-15.2015

Copyright $\odot 2015$ the authors $\quad 0270-6474 / 15 / 3514002-07 \$ 15.00 / 0$ 
a

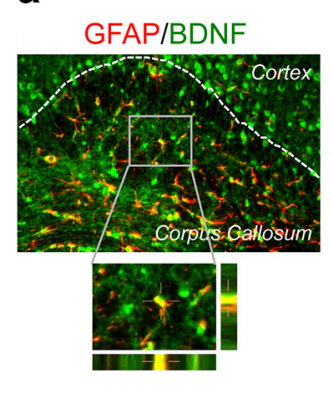
Sham

e

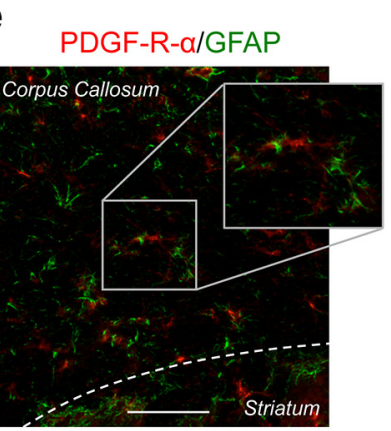

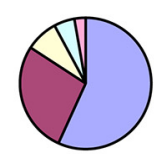

BCAS

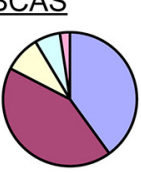

f
C

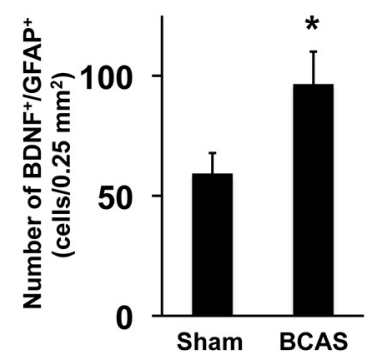

g

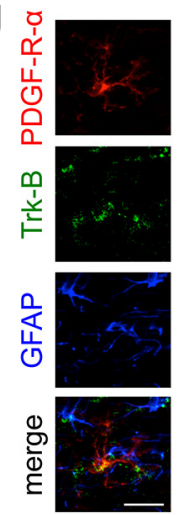

d

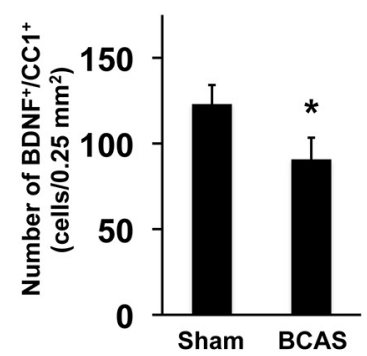

h

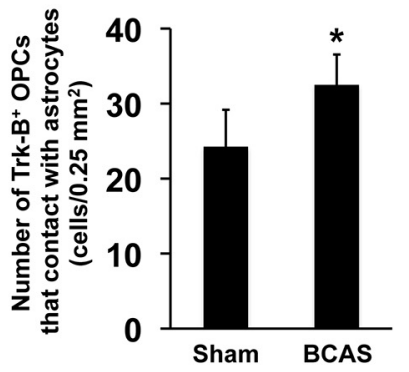

Figure 1. Astrocytes produce BDNF in cerebral white matter in mice. $\boldsymbol{a}$, Representative images of BDNF (green) and GFAP (astrocyte marker; red) immunostaining in mouse corpus callosum. Z-stack images confirmed that astrocytes produced BDNF. $\boldsymbol{b}$, Two-month-old male mice were subjected to sham or BCAS surgery and brains were taken out at day 28 . Immunohistochemistry using anti-BDNF antibody along with cell-specific marker antibodies (CC1: oligodendrocyte, GFAP: astrocyte, lectin: endothelium, PDGF-R- $\alpha$ : OPC, NeuN: neuron) showed that oligodendrocytes and astrocytes were the major cell types for BDNF production in mouse corpus callosum. The pie charts were generated based on the average of numbers for BDNF ${ }^{+}$cells in corpus callosum. $n=5 . c_{\text {, }}$ $\boldsymbol{d}$, Histogram showing the number of BDNF/GFAP-double-positive cells and BDNF/CC1-double-positive cells in mouse corpus callosum. Data are mean \pm SD of $n=5$. ${ }^{*} p<0.05$. $\boldsymbol{e}$, Representative images of PDGF-R- $\alpha$ (OPC marker; red) and GFAP (astrocyte marker; green) immunostaining in cerebral corpus callosum of 2 months old mice. Scale bar, $100 \mu m$. $f$, Electron micrograph showing that OPCs attach to astrocytes in 2-month-old rats. Arrowheads indicate PDGF-R- $\alpha{ }^{+}$signals. BL, Basal lamina; EC, endothelial cell; Astro, astrocytes. Scale bar, 500 nm. $\boldsymbol{g}$, Representative images of PDGF-R- $\alpha$ (OPC marker; red), Trk-B (high affinity BDNF receptor; green), and GFAP (astrocyte marker; blue) immunostaining in cerebral corpus callosum of 2-month-old mice. OPCs that expressed Trk-B were located in close proximity to astrocytes. Scale bar, $50 \mu \mathrm{m}$. $\boldsymbol{h}$, Histogram showing the number of Trk-B ${ }^{+}$OPCs that showed close contact with astrocytes cells in mouse corpus callosum. Data are mean \pm SD of $n=5 .{ }^{*} p<0.05$.

In addition to these roles in sustaining homeostasis, OPCs may also play an essential role in repairing white matter after injury. After stroke or white matter injury, enhanced proliferation, migration, and differentiation of OPCs are observed in damaged and recovering white matter regions (Mandai et al., 1997; Gregersen et al., 2001). Trophic factors may be involved in oligodendrocyte regeneration (oligodendrogenesis) after injury because OPCs express many types of growth factor receptors. In particular, brain-derived neurotrophic factor (BDNF) is believed to be a key factor for OPCs. Several studies have now confirmed that BDNF regulates the function of oligodendrocyte lineage cells. For example, BDNF promotes OPC proliferation and differentiation into mature oligodendrocytes via the Trk-B receptor both in vitro and in vivo (Van't Veer et al., 2009; Vondran et al., 2010; Xiao et al., 2010; Xiao et al., 2012). BDNF may also work on neural stem/progenitor cells to trigger their differentiation into oligodendrocyte lineage cells (Chen et al., 2013). Moreover, studies using BDNF knock-out animals demonstrated that BDNF promotes the proliferation and differentiation of OPCs and is required in normal CNS myelination (Cellerino et al., 1997; Xiao et al., 2010).

Together, this background knowledge suggests that BDNF may play a role in OPC-mediated recovery after white matter damage, but what types of cells secrete BDNF to support OPC maturation and white matter recovery? Because astrocytes are known to be a major type of secretory support cell in the CNS and because they comprise a major component of the reactive gliotic response after brain injury, it is attractive to hypothesize a phenomenon of astrocyte-OPC trophic coupling, whereby reactive astrocytes may in fact produce BDNF to promote recovery after white matter injury. In this study, we combined in vitro cell culture experiments and the astrocyte-specific BDNF knock-down mouse model to assess this hypothesis of an astrocyte-OPC trophic niche for oligodendrogenesis.

\section{Materials and Methods}

Cerebral prolonged hypoperfusion model. All experiments were performed following an institutionally approved protocol in accordance with the National Institutes of Health Guide for the Care and Use of Laboratory Animals. For inducing cerebral chronic hypoperfusion stress, a microcoil (0.18 mm diameter; Sawane Spring) was applied to bilateral common carotid arteries of male C57BL/6 mice (10 weeks old; Charles River Laboratories). All in vivo experiments and measurements were performed in a blinded and randomized manner. Animal numbers for each experiment are described in the figure legends.

$O P C$ differentiation assay in vivo. Mice were intraperitoneally injected with $50 \mathrm{mg} / \mathrm{kg}$ bromodeoxyuridine (BrdU; Sigma-Aldrich) twice a day with a $12 \mathrm{~h}$ interval between 5 and $14 \mathrm{~d}$ after the surgery. The mice were killed $28 \mathrm{~d}$ after the surgery.

Immunohistochemistry. Sixteen-micrometer-thick consecutive coronal sections from PFA-fixed brains were used. Primary antibodies used in this study were as follows: anti-BDNF (1:100; Abcam), anti-TrkB (against extracellular domain of full-length form; 1:100; Abcam), anti- 

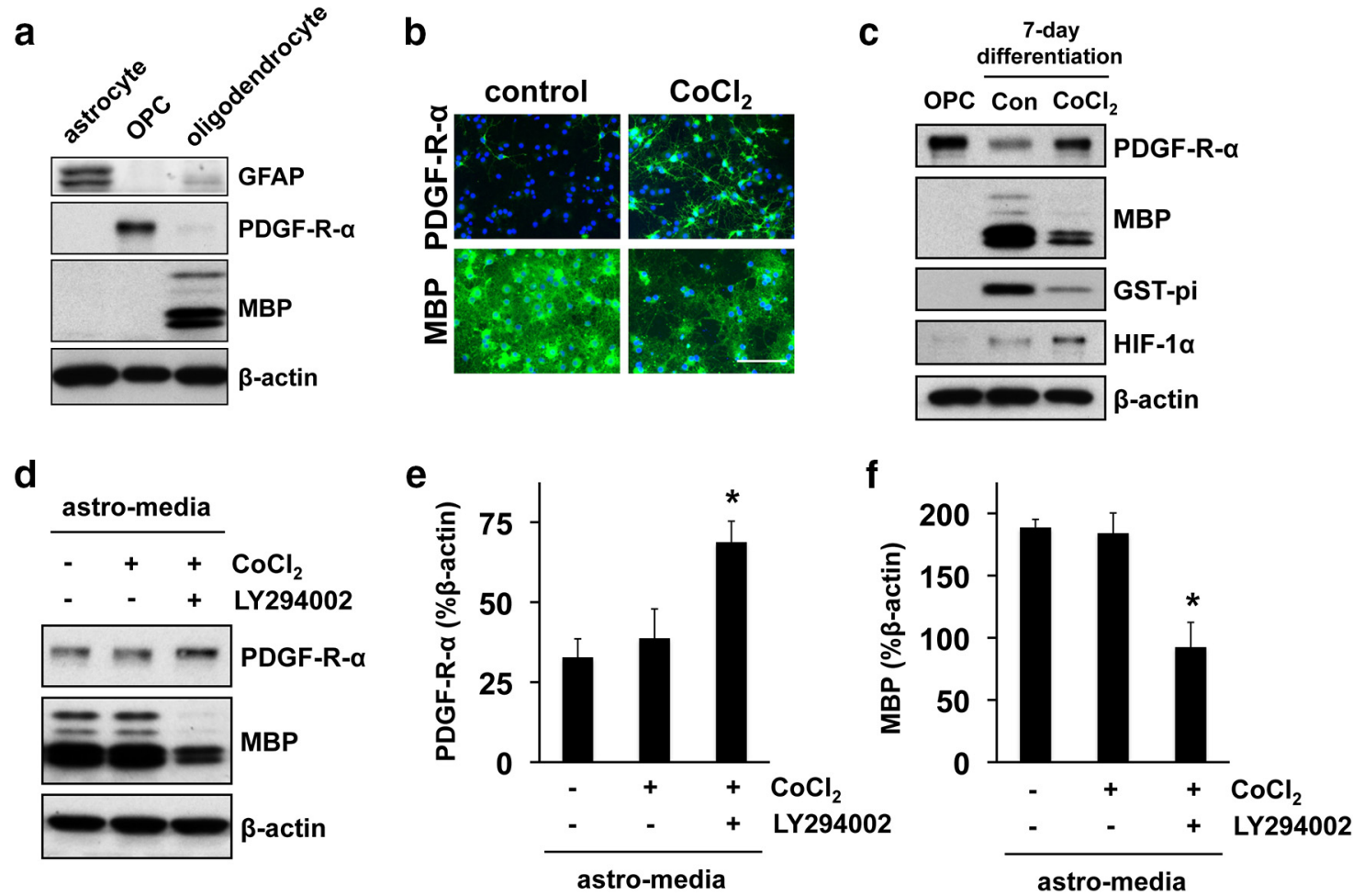

Figure 2. Astrocytes promote $\mathrm{OPC}$ maturation under diseased conditions in vitro. $\boldsymbol{a}$, Western blotting confirmed that our cultured astrocytes, $\mathrm{OPC}$, and oligodendrocytes were positive for their specific markers (GFAP for astrocytes, PDGF-R- $\alpha$ for OPCS, MBP for oligodendrocytes). $\boldsymbol{b}$, Representative images of PDGF-R- $\alpha$ (OPC marker; green) and MBP (oligodendrocyte marker; green) immunostaining in oligodendrocyte cultures. Cell nucleus was stained with DAPI (blue). Scale bar, $50 \mu \mathrm{m}$. c, Representative images of PDGF-R- $\alpha$ (OPC marker), MBP (oligodendrocyte marker), and GST- $\pi$ (oligodendrocyte marker) Western blotting in OPC cultures. OPCs were positive for PDGF-R- $\alpha$ but not for MBP/GST- $\pi$. After culture in OPC differentiation medium for $7 \mathrm{~d}, 0 \mathrm{PCs}$ differentiated into mature oligodendrocytes that expressed MBP/GST- $\pi$ but not PDGF-R- $\alpha$. However, cells with $\mathrm{CoCl}_{2}$ treatment did not differentiate into mature oligodendrocytes. HIF- $1 \alpha$ is a marker for hypoxic conditions and $\beta$-actin is an internal control. Con stands for control. $\boldsymbol{d}-\boldsymbol{f}$, Cell lysates from OPC cultures were subjected to Western blotting. OPCs were maintained for $7 \mathrm{~d}$ in OPC differentiation medium containing astro-media with or without $\mathrm{COCl}_{2}(1 \mu \mathrm{M})$ and $\mathrm{LY} 294002$ (1 $\mu \mathrm{M}$; Pl3K inhibitor). Astro-medium supported the OPC maturation even under hypoxic conditions, but $\mathrm{LY} 294002$ canceled the OPC supportive effects of astro-medium. Data are mean \pm SD of $n=4 .{ }^{*} p<0.05$.

PDGFR- $\alpha$ (1:100; R\&D Systems), anti-GFAP (1:300; Invitrogen), antiCC-1 (1:100; Calbiochem), anti-GST- $\pi$ (1:100; MBL), anti-NeuN (1: 100; Millipore), anti-CD31 (1:100; BD Biosciences), and anti-BrdU (1: 50; Oxford Biotechnology). An investigator blinded to the experimental groups counted the number of stained cells in the lateral side of the corpus callosum (bregma $+1.18 \mathrm{~mm},+0.98 \mathrm{~mm}$, and $+0.74 \mathrm{~mm}$ ).

Fluoromyelin staining. Twelve-micrometer-thick coronal sections (bregma $+0.86 \mathrm{~mm}$ to $+0.50 \mathrm{~mm}$ ) were incubated with FluoroMyelin Green fluorescent myelin stain (1:300; Molecular Probes) for $20 \mathrm{~min}$ at room temperature. Semiquantification of the intensity of fluoromyelin staining was conducted on $10 \times$ magnification images and viewed using a Nikon upright microscope. Intensity of fluoromyelin staining was analyzed by quantifying mean intensity of the entire field of view for three brain sections of each animal using ImageJ analysis software.

Breeding and genotyping of mutant mice. GFAP ${ }^{\text {Cre }}$ mice were crossed with $\mathrm{BDNF}^{\mathrm{fl} / \mathrm{fl}}$ mice. GFAP ${ }^{\mathrm{Cre}}$ mice and $\mathrm{BDNF}^{\mathrm{fl} / \mathrm{fl}}$ mice were purchased from Jackson Laboratories. Genotyping PCR was conducted according to the manufacturer's instruction.

Cell culture. Primary cortical OPCs and astrocytes were prepared and maintained as described previously (Miyamoto et al., 2013). To differentiate OPCs to myelin basic protein-positive oligodendrocytes, the culture medium was switched to DMEM containing $1 \%$ penicillin/streptomycin, $10 \mathrm{ng} / \mathrm{ml}$ ciliary neurotrophic factor, $15 \mathrm{~nm}$ triiodo-L-thyronine, and 2\% B27 supplement (DMEM). To mimic hypoxic conditions in vitro, cells were incubated with a nonlethal concentration of $\mathrm{CoCl}_{2}$ as described previously (Miyamoto et al., 2013). Hypoxic conditions were confirmed by increase of HIF- $1 \alpha$ expression.

Preparation of astrocyte conditioned medium. Cell cultures were washed with PBS and maintained in basal medium without serum and growth supplement for $24 \mathrm{~h}$. Culture medium was then collected and centrifuged at $10,000 \times g$ for $5 \mathrm{~min}$ at $4^{\circ} \mathrm{C}$ to remove cells and debris. The astrocyte- conditioned medium (astro-medium) was stored at $-80^{\circ} \mathrm{C}$ until use. Astro-medium was filtered with recombinant human TrkB-Fc chimera (R\&D Systems) to remove BDNF, as described previously (Guo et al., 2008).

Immunocytochemistry. Cells were washed with ice-cold PBS, pH 7.4, followed by $4 \%$ PFA for $15 \mathrm{~min}$. After being washed with PBS containing $0.1 \%$ Triton X-100, cells were incubated with $1 \%$ bovine serum albumin in PBS for $1 \mathrm{~h}$. The primary antibodies used in this study were anti-MBP (1:100; Thermo Scientific) and anti-PDGFR- $\alpha$ (1:200; Santa Cruz Biotechnology).

Western blotting. Cells were collected in lysis buffer (Pro-PREP Protein Extraction Kit; iNtRON Biotechnology). The primary antibodies used in this study were as follows: anti-MBP (1:1000; Thermo Scientific), antiHIF1 $\alpha$ (a marker for hypoxic conditions; 1:3000; Abcam), anti-PDGFR $\alpha$ (1:3000; Santa Cruz Biotechnology), anti-GST- $\pi$ (1:5000; MBL International), anti-GFAP (1:1000; BD PharMingen), and anti- $\beta$-actin (1: 10000; Sigma-Aldrich) antibodies.

Statistical analysis. Statistical significance was evaluated using the unpaired $t$ test to compare differences between the two groups and a oneway ANOVA followed by Tukey's honestly significant difference test for multiple comparisons. Data are expressed as mean \pm SD. A $p$ value of $<0.05$ was considered statistically significant.

\section{Results}

After prolonged cerebral hypoperfusion by bilateral common carotid artery stenosis (BCAS), a reduction in oligodendrocyte cell counts and a corresponding increase in astrocyte cell counts were noted in the affected corpus callosum, consistent with the known effects of prolonged white matter hypoperfusion (GFAP-sham: $92.3 \pm 8.3$, GFAP-BCAS: $132.1 \pm 13.1$, CC1-sham: $183.2 \pm 13.9$, 
a

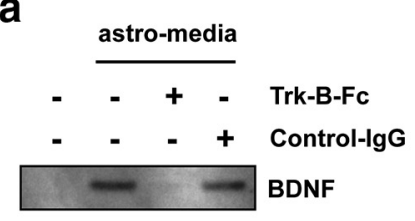

b

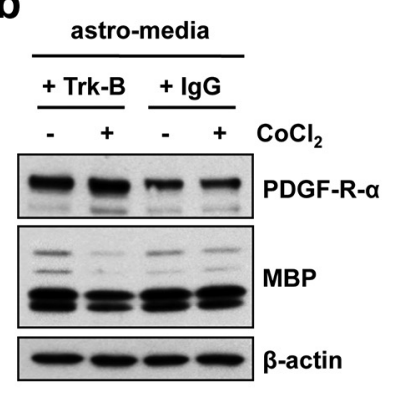

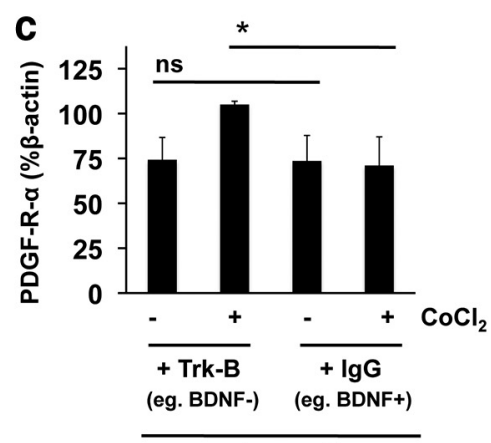

astro-media d

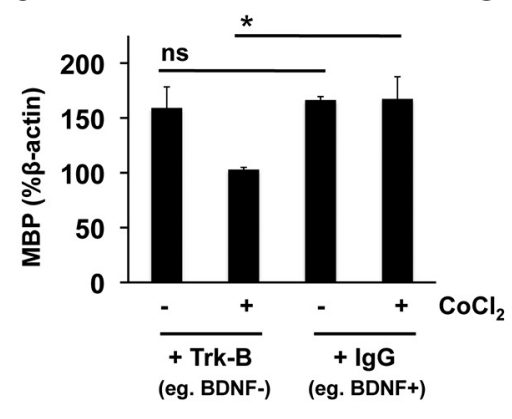

e

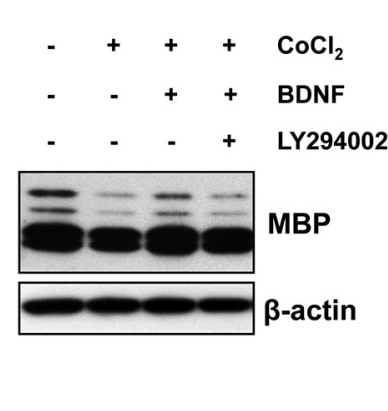

f

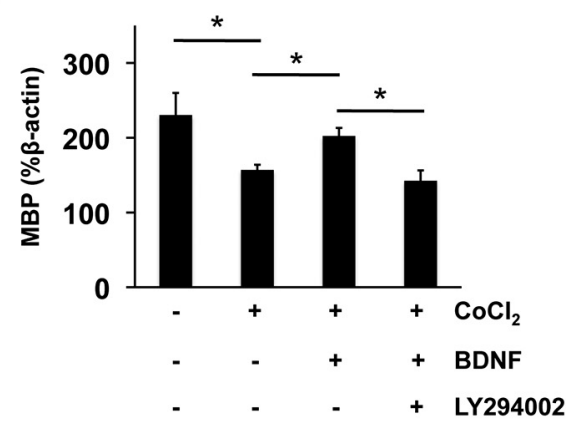

astro-media

Figure 3. Astrocyte-derived BDNF supports OPC maturation under diseased conditions in vitro. $\boldsymbol{a}$, Representative images of BDNF Western blotting in astro-medium. First lane is standard astrocyte culture medium (e.g., DMEM) that does not contain detectable levels of BDNF. Second lane is astro-medium. Third and fourth lanes are astro-media that were filtered with Trk-B-Fc or control-lgG, respectively. $\boldsymbol{b}-\boldsymbol{d}$, Cell lysates from OPC cultures were subjected to Western blotting. OPCs were maintained for $7 \mathrm{~d}$ in OPC differentiation medium containing astro-medium with or without $\mathrm{CoCl}_{2}(1 \mu \mathrm{M})$. Astro-medium was prefiltered with Trk-B-Fc or control-IgG. There was no difference in PDGF-R- $\alpha$ and MBP expression levels between normal astro-medium (IgG filtering) and BDNF-deficient astro-medium (Trk-B filtering). However, under the hypoxic conditions, BDNF-deficient astro-medium (Trk-B filtering) did not support OPC maturation. Data are mean \pm SD of $n=$ 3. ${ }^{*} p<0.05$. $e$, $f$, Cell lysates from OPC cultures were subjected to Western blotting. OPCs were maintained for $7 \mathrm{~d}$ in OPC differentiation medium with or without $\mathrm{BDNF}$ (10 nM), $\mathrm{COCl} \mathrm{C}_{2}(1 \mu \mathrm{M})$, and LY294002 $(1 \mu \mathrm{M})$. The hypoxic stress by $\mathrm{CoCl}_{2}$ inhibited the OPC maturation, but BDNF supported the OPC maturation under the hypoxic conditions. The effects of $\mathrm{BDNF}$ in OPC maturation were inhibited by cotreatment with LY294002. Data are mean \pm SD of $n=4 .{ }^{*} p<0.05$.

CC1-BCAS: $157.3 \pm 10.9$ cells $/ 0.25 \mathrm{~mm}^{2}$ ). Based on this model of white matter injury, we first sought to confirm that astrocytes produce BDNF. Immunohistochemistry was performed using anti-BDNF antibody along with cell-specific marker antibodies (CC1: oligodendrocyte, GFAP: astrocyte, lectin: endothelium, PDGF-R- $\alpha$ : OPC, NeuN: neuron). Brain sections from control mice were compared with those from mice with white matter injury due to prolonged cerebral hypoperfusion. $\mathrm{GFAP}^{+}$astrocytes indeed expressed BDNF in corpus callosum (e.g., cerebral white matter; Fig. 1a). Notably, the pattern of BDNF expression changed after white matter damage: the number of BDNF/GFAPdouble-positive cells increased and the number of BDNF/ CC1-double-positive cells decreased (Fig. 1b-d). Importantly, immunostaining (Fig. 1e) and immunoelectron microscopy (Fig. 1f) confirmed that some astrocytes were located in close proximity to OPCs. In addition, the white matter OPCs, which are adjacent to astrocytes, indeed expressed Trk-B (BDNF receptor; Fig. $1 g, h)$. This appearance of $\mathrm{BDNF}^{+}$astrocytes, together with $\mathrm{TrkB}^{+}$OPCs, may be consistent with our hypothesis of trophic coupling between these two cell types within recovering white matter.

To determine whether astrocytic BDNF supports OPC maturation, we then conducted in vitro medium transfer experiments. Cultured astrocytes and OPCs were obtained from neonatal rat cortex and cultured separately. The purity of our astrocytes and OPCs were confirmed with cell-type-specific markers by Western blotting (Fig. 2a). In addition, Western blotting confirmed that PDGF-R- $\alpha^{+}$OPCs can be successfully differentiated into myelin-basic-protein-positive $\left(\mathrm{MBP}^{+}\right)$oligodendrocytes at day 7 after switching from OPC culture media to OPC differentiation medium (Fig. 2a). When the OPCs were subjected to prolonged hypoxic stress by exposure to sublethal $\mathrm{CoCl}_{2}$ for $7 \mathrm{~d}$ in the OPC differentiation medium, in vitro OPC maturation was suppressed (Fig. 2b,c), but when OPCs were incubated in astro-medium, OPCs were successfully differentiated into $\mathrm{MBP}^{+}$oligodendrocytes even under hypoxic stress conditions (Fig. $2 d-f$ ). This phenomenon may involve PI3K/Akt signaling because the ability of astro-medium to rescue OPC differentiation was cancelled by the PI3K inhibitor LY294002 (Fig. 2d-f).

Next, to determine whether astrocytic BDNF is truly an important factor for astro-medium-induced OPC maturation under stressed conditions, we removed BDNF from astro-medium by filtering with Trk-B-Fc. The standard culture medium (e.g., DMEM) for astrocytes did not include BDNF at the level detectable by Western blot (Fig. $3 a$, first lane), but the astro-medium contained a substantial level of BDNF (Fig. $3 a$, second lane). When astro-medium was incubated with Trk-B-Fc, BDNF was successfully filtered out (Fig. $3 a$, third lane); however, control IgG did not filter out BDNF (Fig. $3 a$, fourth lane). We then compared the effects on OPC maturation for normal astro-medium (IgG filtration) versus BDNF-deficient astro-medium (Trk-B-Fc filtration). Under normal uninjured conditions $(0 \mu \mathrm{M} \mathrm{CoCl})_{2}$, OPC cultures had similar levels of MBP expression in normal astro-medium and in BDNF-deficient astro-medium (Fig. $3 b-d$ ). However, under hypoxic stress conditions induced by $1 \mu \mathrm{M}$ $\mathrm{CoCl}_{2}, \mathrm{OPCs}$ exhibited a lower level of MBP expression in BDNF- 
a

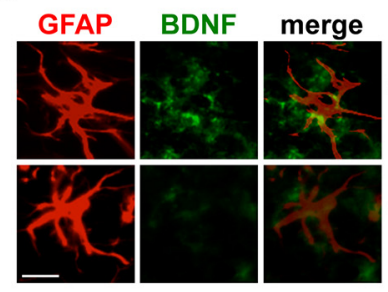

GFAPwt/BDNFflox/wt (wild type mice)

GFAPcre/BDNFflox/wt (transgenic mice) b

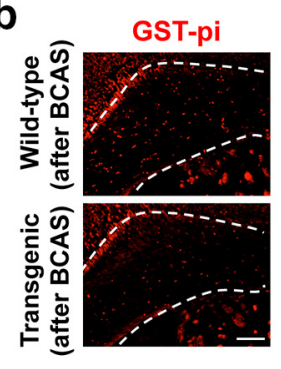

C

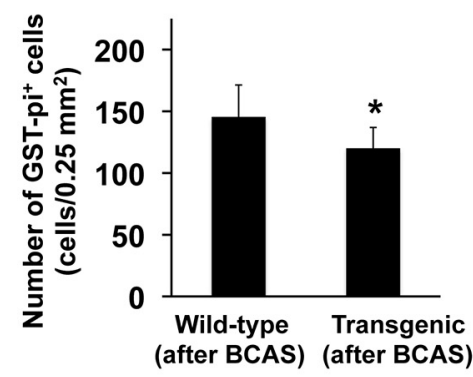

\section{f}

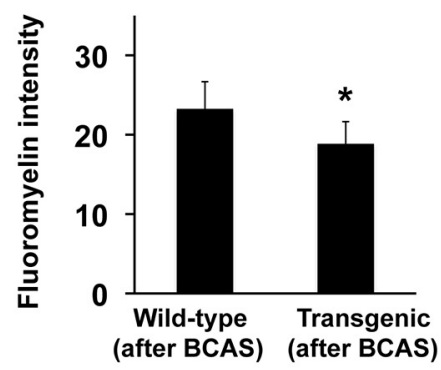

Figure 4. Astrocyte-derived BDNF supports oligodendrogenesis after white matter injury in mice. $\boldsymbol{a}$, Representative images of GFAP (astrocyte marker; red) and BDNF (green) immunostaining in corpus callosum of GFAP ${ }^{\mathrm{wt}} / \mathrm{BDNF}^{\mathrm{ff} / \mathrm{wt}}$ (wild-type) and GFAP ${ }^{\mathrm{cre}} / \mathrm{BDNF}^{\mathrm{ff} / \mathrm{wt}}$ (transgenic) mice. BDNF expression was downregulated in astrocytes in transgenic mice. Scale bar, $20 \mu \mathrm{m} . \boldsymbol{b}, \boldsymbol{c}$, Immunostaining of GST- $\pi$ (oligodendrocyte marker) in mouse corpus callosum. Wild-type $(n=6)$ and transgenic $(n=5)$ mice were subjected to prolonged cerebral hypoperfusion by BCAS. At day 28, brains were taken out for immunohistochemistry. Transgenic mice showed lower number of GST- $\pi^{+}$cells. Scale bar, $100 \mu \mathrm{m}$. Data are mean \pm SD. ${ }^{*} p<0.05$. $\boldsymbol{d}, \boldsymbol{e}$, In vivo OPC maturation assay. Wild-type $(n=6)$ and transgenic $(n=5)$ mice were subjected to prolonged cerebral hypoperfusion by BCAS. Mice were treated with BrdU between days 5 and 14 . Transgenic mice showed a lower number of GST- $\pi$ /BrdU-double-positive cells (i.e., newly generated oligodendrocytes) at day 28 . Control mice $($ no BCAS, $n=6$ ) showed the baseline of oligodendrocyte renewal in mouse corpus callosum under normal conditions. Scale bar, $100 \mu \mathrm{m}$. Data are mean \pm SD. ${ }^{*} p<0.05$. $\boldsymbol{f}$, Fluoromyelin staining showed that myelin density in corpus callosum region at day 28 in GFAP $^{\text {cre }} /$ BDNF $^{\mathrm{fl} / \mathrm{wt}}$ mice was lower than that in GFAP ${ }^{\mathrm{wt}} / \mathrm{BDNF}^{\mathrm{fl} / \mathrm{wt}}$ mice. Data are mean $\pm \mathrm{SD} .{ }^{*} p<0.05$.

deficient astro-medium compared with normal astro-medium (Fig. $3 b-d$ ). In parallel experiments, we confirmed that recombinant BDNF indeed promoted OPC maturation under diseased conditions in our in vitro culture system (Fig. $3 e, f)$. Together, our in vitro experiments demonstrate that astrocyte-derived BDNF may be a critical factor for OPC maturation under pathological conditions in vitro.

Finally, we investigated whether astrocyte-derived BDNF promotes oligodendrocyte regeneration (oligodendrogenesis) after white matter damage in vivo. For this purpose, we analyzed a transgenic mouse line in which BDNF expression is downregulated specifically in astrocytes (Fig. 4a). Immunostaining of mouse brain sections showed that the transgenic $\left(\mathrm{GFAP}^{\mathrm{cre}} / \mathrm{BDNF}^{\mathrm{wt} / \mathrm{fl}}\right)$ mice did not show significant loss of GST- $\pi^{+}$oligodendrocytes compared with wild-type (GFAP ${ }^{\mathrm{wt}} /$ $\mathrm{BDNF}^{\mathrm{wt} / \mathrm{fl}}$ ) mice under normal conditions (wt mice: $177.5 \pm 15.9$ vs tg mice: $176.5 \pm 8.8$ cells $/ 0.25 \mathrm{~mm}^{2}$ ). Then, we subjected both wild-type and transgenic mice to prolonged cerebral hypoperfusion by bilateral common carotid artery stenosis for $28 \mathrm{~d}$. Immunostaining showed that transgenic mice with downregulated BDNF expression in astrocytes showed a lower number of mature oligodendrocytes (GST- $\pi^{+}$cells) in the corpus callosum compared with wild-type mice (Fig. $4 b, c)$. Notably, an assessment of OPC maturation in vivo by BrdU injection/staining demonstrated that in the transgenic mice, there was a lower number of newly generated oligodendrocytes (BrdU/GST- $\pi$-doublepositive cells) than wild-type mice after $28 \mathrm{~d}$ of cerebral hypoperfusion (Fig. 4d,e). Conversely, there were no significant differences in the numbers of proliferating OPCs (e.g., Ki $67^{+}$ PDGF-R- $\alpha$ cells; wt mice: $11.6 \pm 3.0$ vs tg mice: $8.4 \pm 3.8$ cells/ $\mathrm{mm}^{2}$ ) and damaged OPCs (e.g., ssDNA ${ }^{+}$PDGF-R- $\alpha$ cells; wt mice: $25.6 \pm 3.8$ vs tg mice: $30.8 \pm 8.4$ cells $/ \mathrm{mm}^{2}$ ) on day 28 after cerebral hypoperfusion. Finally, fluoromyelin staining confirmed that myelin density in the transgenic mice was also lower than that in wild-type mice (Fig. $4 f$ ).

\section{Discussion}

Almost 50\% of the human brain is composed of white matter (Zhang and Sejnowski, 2000) and white matter damage is a clinically important aspect of several CNS diseases, including stroke and vascular dementia. During the acute phase of injury, damage to oligodendrocytes leads to white matter dysfunction through the loss of myelin. Thereafter, as the damaged brain attempts to recover, OPCs may provide an endogenous mechanism for reconstituting oligodendrocyte pools and myelin integrity and function. In this study, our in vitro and in vivo data show that, after white matter injury, astrocytes produce BDNF to help OPCs differentiate into mature oligodendrocytes to repair white matter damage. This non-cell-autonomous mechanism in oligodendrogenesis and remyelination may be vital for sustaining white matter homeostasis after ischemic injury or disease.

Astrocytes are highly secretory cells and communicate with neighboring cells by exchanging various trophic factors. In addition to their traditional roles in supporting neurotransmission, astrocytes have been shown to influence oligodendrocyte lineage cells positively or negatively through releasing multiple trophic factors. Therefore, we hypothesized that, after white matter damage, astrocytes support oligodendrocyte regeneration by releasing BDNF to promote the differentiation of residual OPCs. Our findings may be consistent with two recent studies in this field. In experimentally induced subcortical stroke in rats, intravenous injection of exogenous 
BDNF was associated with increased OPC proliferation and remyelination, as well as improvement in functional outcome (Ramos-Cejudo et al., 2015). Furthermore, in a cuprizoneinduced demyelination mouse model, BDNF from reactive astrocytes helped to restore myelin integrity (Fulmer et al., 2014). This later study may be especially important because it lays the groundwork for our own investigations; BDNFimmunopositive astrocytes can assist remyelination, but what is the mechanism underlying this recovery phenomenon? By combining gain- and loss-of-function experiments in cell culture models and a cell-specific transgenic mouse model, we showed a direct effect of astrocyte-secreted BDNF on OPC maturation, oligodendrocyte replacement, and white matter remyelination.

Together, our findings support the notion of a trophic coupling niche between astrocytes and OPCs in damaged white matter. However, there are some important caveats to keep in mind. First, we demonstrate that astrocytes play an important role in OPC maturation after injury, but other cell types may also contribute to OPC function. In fact, our data showed that oligodendrocytes and cerebral endothelial cells are also important cell types in secreting BDNF in corpus callosum, although to a lesser extent than astrocytes. Future studies are needed to examine carefully how oligodendrocyte- or endothelium-derived BDNF contributes to OPC function under white matter pathology. Second, we did not assess neurological and/or cognitive function in our current study. However, neurological deficits in this model are well established, including sensorimotor and memory deficits, and white matter lesions are known to be negatively correlated with cognitive and behavioral function (Ihara et al., 2014). How astrocyte-derived BDNF alleviates behavioral deficits after white matter dysfunction would be an important research topic in the future. Third, our cell culture approach in comparing immature OPC (PDGF-R- $\alpha$ ) versus mature oligodendrocyte markers (MBP or GST- $\pi$ ) to assess in vitro OPC maturation is now relatively commonly used. However, we should be careful with these Western blot markers because cells may change their protein production profiles in unexpected ways. More mechanistic studies into how BDNF signaling modulates discrete steps in OPC maturation would be informative. Fourth, although mice with BDNF downregulation in astrocytes exhibited significant changes in newly generated oligodendrocytes and myelin density, the difference in oligodendrogenesis between wild-type and the transgenic mice was not huge. Because previous studies demonstrated that full deletion of BDNF may cause severe oligodendrocyte dysfunction at baseline (Korte et al., 1995; Djalali et al., 2005), we conducted experiments using GFAPcre conditional BDNF heterozygous mice first. Further, the heterozygous mice exhibited lower oligodendrogenesis after injury, which may support our hypothesis. Nevertheless, in the future, analyzing GFAPcre conditional total BDNF KO mice would be still important to supplement our current findings. Fifth, our cell culture experiments provided direct evidence that astrocytic BDNF promoted OPC maturation and our in vivo data showed that transgenic mice with BDNF downregulation in astrocytes exhibited less oligodendrogenesis. Furthermore, in this cerebral hypoperfusion model, there is no significant neuronal damage per se; that is, the majority of the tissue damage involves white matter injury in the form of demyelination (Shibata et al., 2004). Therefore, at least within the limited context of our model systems, it would be reasonable to think that astrocyte-derived BDNF may directly affect OPCs. However, we cannot unequivocally rule out the possibility that astrocytic-BDNF may also indirectly promote OPC maturation via influences on neuronal survival and function. Future studies are warranted to dissect multiple-cell interactions among astrocytes, OPCs, neurons, and axons in various diseased conditions. Finally, reactive astrocytes may compensate for white matter damage by secreting BDNF to support oligodendrogenesis under the prolonged cerebral hypoperfusion conditions. However, eventually, those mice with hypoperfusion stress would exhibit significant oligodendrocyte loss and myelin degradation over time. Under pathological conditions, endogenous astrocyte-derived BDNF may not be sufficient to overwhelm these deleterious cascades. If this is true, future studies are needed to determine whether overexpression of BDNF in astrocytes would protect white matter against hypoxic stress in vivo.

In conclusion, our current study demonstrates a novel noncell-autonomous mechanism in oligodendrocyte regeneration after white matter injury. After white matter damage associated with prolonged cerebral hypoperfusion, reactive astrocytes produce more BDNF, which activates repair processes and accelerate endogenous oligodendrogenesis to ease white matter damage. Insofar as hypoperfusion may damage white matter in stroke and vascular dementia, regulating astrocytic BDNF expression may provide a broad therapeutic approach for cerebrovascular disorders.

\section{References}

Cellerino A, Carroll P, Thoenen H, Barde YA (1997) Reduced size of retinal ganglion cell axons and hypomyelination in mice lacking brain-derived neurotrophic factor. Mol Cell Neurosci 9:397-408. CrossRef Medline

Chen BY, Wang X, Wang ZY, Wang YZ, Chen LW, Luo ZJ (2013) Brainderived neurotrophic factor stimulates proliferation and differentiation of neural stem cells, possibly by triggering the Wnt/beta-catenin signaling pathway. J Neurosci Res 91:30-41. Medline

Dawson MR, Polito A, Levine JM, Reynolds R (2003) NG2-expressing glial progenitor cells: an abundant and widespread population of cycling cells in the adult rat CNS. Mol Cell Neurosci 24:476-488. CrossRef Medline

Djalali S, Höltje M, Grosse G, Rothe T, Stroh T, Grosse J, Deng DR, Hellweg R, Grantyn R, Hörtnagl H, Ahnert-Hilger G (2005) Effects of brainderived neurotrophic factor (BDNF) on glial cells and serotonergic neurones during development. J Neurochem 92:616-627. CrossRef Medline

Fulmer CG, VonDran MW, Stillman AA, Huang Y, Hempstead BL, Dreyfus CF (2014) Astrocyte-derived BDNF supports myelin protein synthesis after cuprizone-induced demyelination. J Neurosci 34:8186-8196. CrossRef Medline

Gregersen R, Christensen T, Lehrmann E, Diemer NH, Finsen B (2001) Focal cerebral ischemia induces increased myelin basic protein and growthassociated protein- 43 gene transcription in peri-infarct areas in the rat brain. Exp Brain Res 138:384-392. CrossRef Medline

Guo S, Kim WJ, Lok J, Lee SR, Besancon E, Luo BH, Stins MF, Wang X, Dedhar S, Lo EH (2008) Neuroprotection via matrix-trophic coupling between cerebral endothelial cells and neurons. Proc Natl Acad Sci U S A 105:7582-7587. CrossRef Medline

Ihara M, Taguchi A, Maki T, Washida K, Tomimoto H (2014) A mouse model of chronic cerebral hypoperfusion characterizing features of vascular cognitive impairment. Methods Mol Biol 1135:95-102. CrossRef Medline

Korte M, Carroll P, Wolf E, Brem G, Thoenen H, Bonhoeffer T (1995) Hippocampal long-term potentiation is impaired in mice lacking brainderived neurotrophic factor. Proc Natl Acad Sci U S A 92:8856-8860. CrossRef Medline

Levine JM, Reynolds R, Fawcett JW (2001) The oligodendrocyte precursor cell in health and disease. Trends Neurosci 24:39-47. Medline

Mandai K, Matsumoto M, Kitagawa K, Matsushita K, Ohtsuki T, Mabuchi T, Colman DR, Kamada T, Yanagihara T (1997) Ischemic damage and 
subsequent proliferation of oligodendrocytes in focal cerebral ischemia. Neuroscience 77:849-861. CrossRef Medline

Miyamoto N, Pham LD, Hayakawa K, Matsuzaki T, Seo JH, Magnain C, Ayata C, Kim KW, Boas D, Lo EH, Arai K (2013) Age-related decline in oligodendrogenesis retards white matter repair in mice. Stroke 44:2573-2578. CrossRef Medline

Ramos-Cejudo J, Gutiérrez-Fernández M, Otero-Ortega L, Rodríguez-Frutos B, Fuentes B, Vallejo-Cremades MT, Hernanz TN, Cerdán S, DíezTejedor E (2015) Brain-derived neurotrophic factor administration mediated oligodendrocyte differentiation and myelin formation in subcortical ischemic stroke. Stroke 46:221-228. CrossRef Medline

Shibata M, Ohtani R, Ihara M, Tomimoto H (2004) White matter lesions and glial activation in a novel mouse model of chronic cerebral hypoperfusion. Stroke 35:2598-2603. CrossRef Medline

Van't Veer A, Du Y, Fischer TZ, Boetig DR, Wood MR, Dreyfus CF (2009) Brain-derived neurotrophic factor effects on oligodendrocyte progenitors of the basal forebrain are mediated through trkB and the MAP kinase pathway. J Neurosci Res 87:69-78. CrossRef Medline

Vondran MW, Clinton-Luke P, Honeywell JZ, Dreyfus CF (2010) $\mathrm{BDNF}+/$ - mice exhibit deficits in oligodendrocyte lineage cells of the basal forebrain. Glia 58:848-856. Medline

Xiao J, Wong AW, Willingham MM, van den Buuse M, Kilpatrick TJ, Murray SS (2010) Brain-derived neurotrophic factor promotes central nervous system myelination via a direct effect upon oligodendrocytes. Neurosignals 18:186-202. CrossRef Medline

Xiao J, Ferner AH, Wong AW, Denham M, Kilpatrick TJ, Murray SS (2012) Extracellular signal-regulated kinase 1/2 signaling promotes oligodendrocyte myelination in vitro. J Neurochem 122:1167-1180. CrossRef Medline

Zhang K, Sejnowski TJ (2000) A universal scaling law between gray matter and white matter of cerebral cortex. Proc Natl Acad Sci U S A 97:56215626. CrossRef Medline 